\title{
Encapsulation Technology: Opportunity to develop Novel Drug Delivery Systems
}

\section{Jagadevappa Patil}

VT's Shivajirao S Jondhle College of Pharmacy, Asangaon-421 601, Shahapur, Thane, Maharashtra, India

*Corresponding author: Jagadevappa Patil, VT's Shivajirao S Jondhle College of Pharmacy, Asangaon-412 601, Thane, Maharashtra, India, Tel: 9448816812; E-mail: pharmajspatil@gmail.com

Received date: June 03, 2016; Accepted date: June 05, 2016; Published date: June 10, 2016

Copyright: (c) 2016 Patil J. This is an open-access article distributed under the terms of the Creative Commons Attribution License, which permits unrestricted use, distribution, and reproduction in any medium, provided the original author and source are credited.

\section{Introduction}

Microencapsulation is an advanced technology has widely been used in different industries for numerous applications such as graphics, food and agriculture. Drug encapsulation based on microencapsulation technique is a novel approach to design and development of particulate drug delivery systems has also been extensively utilized to solve various pharmaceutical and medicinal problems. In the past few decades, significant advances of fundamental and relevance researches have taken place in the field of pharmaceutics. The recently recognized research thrust on Nano- and microencapsulation is anticipated to have an innovator bang on the human health. To reach this point, substantial attention has been devoted towards the development of novel drug delivery systems with the aim to exclusively target the drug to a specific biological site in order to release at a controlled rate and at the desired time.

\section{Encapsulation Technique}

Microencapsulation process is adopted not only to design sustained release of drug, but also to protect the drug molecule from adverse environment, stabilization, avoid incompatibilities, and mask the unpleasant taste and odor. Microparticles obtained by this technique are widely used for the development of controlled or prolonged release dosage forms. These drug delivery devices also play vital role in improving bioavailability of original drugs with minimum adverse effects1. Literal meaning of the term microencapsulation is used to assign a sort of techniques utilized to entrap solids, liquids or gases into a polymeric matrix or shell to achieve the various advantages enlisted above. Particle formation in this technique is a single step process when compared to alternative film coating procedure. Routinely, this technique involves encapsulation of therapeutic molecule into biocompatible and biodegradable polymer matrix to get particulate mass ranging in the micron size.

\section{Drug Release Mechanisms}

\section{There are many ways identified as mechanism of drug release from encapsulated matrix, the following are few among}

Degradation controlled mechanism: According to this mechanism the drug is first dissolved in polymer matrix and is dispersed homogeneously throughout, and is released on degradation of the matrix. As the degradation of polymer matrix takes place, drug release will be initiated by diffusion.

Diffusion controlled mechanism: In this system, drug release begins with diffusion through polymer matrix membrane prior to or concurrent with the polymer degradation. The drug release rate depends on type of polymer degradation mechanism.

Diffusion controlled reservoir system: According to this mechanism, drug release takes place by diffusion through rate controlling membrane surrounded the polymer matrix and finally the membrane will get eroded after drug delivery process completes. Hence, the drug release by this mechanism will not be affected by degradation of polymer matrix.

\section{Erosion}

Here, the drug release takes place by erosion of polymeric coat membrane/matrix due to adverse $\mathrm{pH}$ and enzymatic hydrolysis [2].

\section{Applications}

- This novel technology has numerous applications in different fields, some are enlisted here.

- Cell immobilization: In plant cell cultures, Human tissue is turned into bio-artificial organs, in continuous fermentation processes.

- In the beverage production, in textiles as a means of imparting finishes and for the protection of liquid crystals.

- Protection of molecules from other compounds.

- Drug delivery: Major application of encapsulation is for developing controlled/sustained drug delivery. This system helps to replace the therapeutic agents which cannot taken by oral route like insulin, gene therapy, and effective delivery of vaccines for treating AIDS, tumors, cancer and diabetes.

- Quality and safety in food, agricultural and environmental sectors. The technique is mainly used in the area of crop protection and Soil inoculation. The technology is utilized to incorporate minerals, vitamins, flavours and essential oils into food materials. In addition, this technique is used to convert liquids to solid powders in the food manufacturing process. This helps to decrease the production costs by allowing batch processing using low cost, powder handling equipment.

\section{Conclusion}

This technique started as a way of encapsulating dyes and flavors, has now become one of the most fascinating fields in the area of novel controlled drug delivery systems. These techniques have been highly developed to such a level that not only micro molecules but also macromolecules such as proteins and genes as micro particulate carriers. Microencapsulation technology is both an art and a science deals with diverse fields. Although the technological advancements facilitated to commercialize several particulate drug delivery products 
Citation: Patil J (2016) Encapsulation Technology: Opportunity to develop Novel Drug Delivery Systems. J Pharmacovigil 4: e157. doi: 10.4172/2329-6887.1000e157

Page 2 of 2

many technical hurdles are to be solved yet. The research in this field has gigantic prospective to give raw materials advantageous character resulting in better products. From time to time new developments in this field have led to new products such as carbonless copy paper followed by controlled release of drugs. Presently, the paper-like displays, self- healing structures and chemical decontaminating fabrics are in receipt of much awareness in microencapsulation technology.

\section{References}

1. Simon B (2005) Microencapsulation: Methods and Industrial Application.

2. Packhauser C, Seidel N, Schnieders J, Kissel T, Maretschek S (2005) Techniques for Parenteral Depot Systems and T heir Application in the Pharmaceutical Industry 4: 99-122.

3. James S [1934] Encylopedia of Pharmaceutical Techonology. 3: 1325-1333. 